\title{
All-optical switching based on optical control of energy transfer between thin-film layers
}

\author{
David S. Bradshaw and David L. Andrews* \\ Nanostructures and Photomolecular Systems, School of Chemical Sciences, \\ University of East Anglia, Norwich NR4 7TJ, United Kingdom
}

\begin{abstract}
The migration of electronic excitation energy between individual particles is a well-studied phenomenon. The ability to exert optical control over this transfer of energy is the subject of much recent research, and it forms the basis for a potential all-optical switching device. In detail, near-field energy transfer from an excited donor nanoparticle (following previous light absorption) to an acceptor particle can, under suitable conditions, be activated or deactivated by the input of a non-resonant laser beam, i.e. optical switching action occurs. It is envisaged that an all-optical device utilizing the described mechanism will involve nanoparticles contained within thin-film deposits on a pair of parallel substrates. Nanolithography is the technique offering the best prospects for the deposition and tailoring of nanoparticles within each optically active layer. This paper gives a theoretical analysis of the non-linear response mechanism, termed optically controlled resonance energy transfer (OCRET). The concept of transfer fidelity, signifying the accuracy of mapping input to designated output, is introduced and its key determinants are identified. Analysis shows that, at reasonable levels of laser intensity, cross-talk to unsought destinations can be effectively extinguished. The advantage of constructing these donor and acceptor thin-film layers around an ultra-thin spacer material (which is suitably transparent) is discussed, and potential applications beyond simple switching are outlined, including logic gates and optical buffers.
\end{abstract}

Keywords: Optical switching, thin films, nanoparticles, energy transfer, optical logic, optical buffers, nanofabrication, nonlinear optics

\section{INTRODUCTION}

Although electronic computer processing capabilities continue to rise more or less according to Moore's Law, all-optical systems have a clear potential for much greater bandwidths and speeds - circumventing the bottlenecks that can result from opto-electronic conversion. For many years it has been known that all-optical switching, based on various forms of photonic interaction in which light is controlled by light, is not only technically realizable but has the capacity to revolutionize computing and telecommunications. Not surprisingly, numerous implementation strategies have been entertained, and many are the subject of vigorous ongoing research. These include: semiconductor quantum wells systems, ${ }^{1-4}$ where optical switching is conceived as an exploitation of optical saturation - though an alternative method, based on atomic quantum interference in electromagnetic induced transparency has recently been demonstrated; ${ }^{5-7}$ photonic crystals with cross-waveguide geometries, offering switching action through the optical Kerr effect; ${ }^{8-10}$ surface plasmon polariton media, in which light-induced dielectric modification at an interface affects the transmission of throughput radiation; ${ }^{1-13}$ and various schemes based on films of bacteriorhodopsin, a light harvesting protein with unique non-linear optical properties. ${ }^{14-16}$

The present paper, however, reports an in-depth analysis of a very different, all-optical switching mechanism, based on the optical control of resonance energy transfer (RET) between particles. When RET occurs spontaneously, following the absorption of light, it usually represents the principal process for the intermolecular translation of electronic energy, from the sites of initial optical excitation. ${ }^{17}$ However, under suitable conditions such processes of energy transfer can be activated or deactivated by (non-resonant) optical stimulation. In many features, optical switches based on this principle differ strikingly from the majority of previously proposed schemes. Here, photonic switching is localized in particle pairs rather than ensembles, and the mechanism allows much shorter operating wavelengths to be engaged, i.e. the ultraviolet or visible range, in contrast to the infrared. RET in its simplest form is known as Förster transfer, a well-studied near-field phenomenon that involves relocation of energy from an electronically excited donor $A$,

*david.andrews@physics.org 
to an acceptor $B$ in its ground state. In quantum electrodynamical terms, this transfer is mediated by a virtual photon. By input of an auxiliary laser field, such energy transfer may be enhanced by a non-linear response mechanism termed laser-assisted resonance energy transfer (LARET), whose efficiency scales linearly with the laser intensity. ${ }^{18,19}$ Optically controlled resonance energy transfer (OCRET), the mechanistic basis for the study reported here, is a related process, differing from LARET in that an important configurable condition is applied. This condition requires that the transition dipole moments of the donor and acceptor, and their mutual displacement vector, be mutually perpendicular - thus excluding a Förster process that would otherwise be possible on symmetry and energetic grounds. By application of the off-resonant laser beam, the transfer of energy is activated, effecting all-optical switching action.

The following analysis begins by developing the fundamental electrodynamic theory for the OCRET mechanism, with consideration of a single donor-acceptor pair (Section 2). The investigation is then extended to an arrangement that could be useful for realistic all-optical switches, taking into account the requirement for a multiplicity of such donor-acceptor partners to operate independently, without significant cross-talk. This arrangement is envisaged to involve nanoparticles (array pixels) contained within thin-film deposits on a pair of parallel substrates. The specific geometric configurations to be examined are square-packed and hexagonal-packed configurations (Sections 3 and 4 , respectively). Further implementation issues are addressed in the concluding Section 5.

\section{COUPLING PAIR}

To specifically determine the efficiency of energy transfer for the OCRET mechanism, a time-dependent perturbation theory method is required. OCRET is distinguished by the fact that the quantum amplitude for Förster excitation transfer has a null result - the consequence of an orthogonal triad condition, i.e. $\mathbf{R} \perp \boldsymbol{\mu}_{A} \perp \mu_{B}$ (where $\mathbf{R}$ is the donor-acceptor displacement vector, and $\mu_{A}, \mu_{B}$ are the salient transition dipole moments of the two particles). In the near-field, the quantum amplitude arising from the input auxiliary beam is given by the general expression; ${ }^{20}$

$$
M^{(4)}=\left(\frac{m \hbar c k}{8 \pi \varepsilon_{0}^{2} V R^{3}}\right) e_{i} \bar{e}_{l}\left(\delta_{j k}-3 \hat{R}_{j} \hat{R}_{k}\right)\left(S_{i j}^{A}(k) S_{l k}^{B}(-k)+S_{i j}^{B}(k) S_{l k}^{A}(-k)\right),
$$

where $m$ is the number of photons (proportional to the irradiance of laser input) in the quantization volume $V$, and the implied summation convention for repeated Cartesian tensor indices is employed. Furthermore, $R$ is assumed to be sufficiently small that the difference in optical phase registered by the donor and acceptor, $\exp ( \pm i \mathbf{k} \cdot \mathbf{R})$, is close to unity; also, $\hbar c k$ denotes the energy of each input photon, and $\mathbf{e}$ is the associated unit polarization vector, with an overbar denoting complex conjugation. The latter technicality signifies a generalization of the theory to admit circular or elliptical polarizations - however, since their employment proves to confer no additional advantages over linear polarization, the overbar will henceforth be omitted. Lastly featured in equation (1) are polarizability tensors, for example $S_{i j}^{A}(k)$, which denote a two-photon event at the particle specified by the superscript; one of these events concerns a virtual photon mediating the RET. The latter is illustrated by the Feynman diagrams of Figure 1(a)-(b), within each of which are depicted the four interaction events that necessitate deployment of a fourth-order perturbation treatment, as indicated in (1) by the superscript of $M$. An energy scheme representing this mechanism is illustrated in Fig. 2.

As detailed elsewhere, ${ }^{21}$ a reasonable assumption to simplify equation (1) involves limiting the explicit form of the polarizability tensor to the three most prominent states. These are to be denoted $|0\rangle,|\sigma\rangle,|\alpha\rangle$ for the donor particle and $|0\rangle,|\tau\rangle,|\beta\rangle$ for the acceptor, where $|\alpha\rangle$ and $|\beta\rangle$ are the levels between which energy transfer occurs. Therefore, equation (1) becomes; 


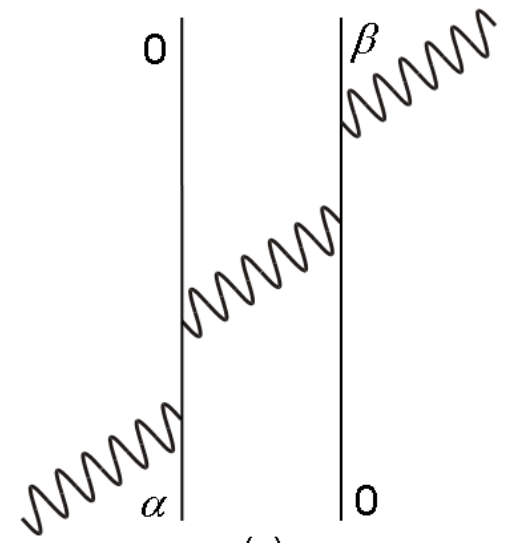

(a)

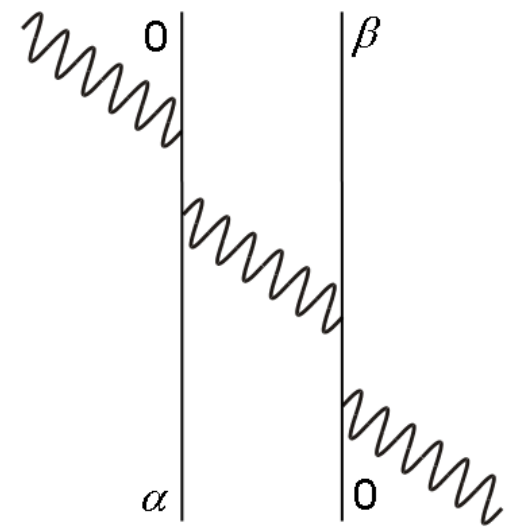

(b)

Figure 1. Typical Feynman diagrams for OCRET. Here, 0 represents a nanoparticle in the ground state; $\alpha$ and $\beta$ relate to the excited state of the donor (on the left) and acceptor (right), respectively. In detail, diagram ( $a$ ) depicts an instantaneous mechanism involving photon absorption and emission at the donor and acceptor, respectively, with a coupling virtual photon created at the donor and annihilated at the acceptor; thus excitation is transferred from $A$ to $B$. Diagram $(b)$ is one of 47 other permutations that will achieve an identical final result. With exclusion of the absorption from and emission to the throughput beam, i.e. only virtual photon transfer between the nanoparticles occurs, the Feynman diagrams for Förster transfer arises.

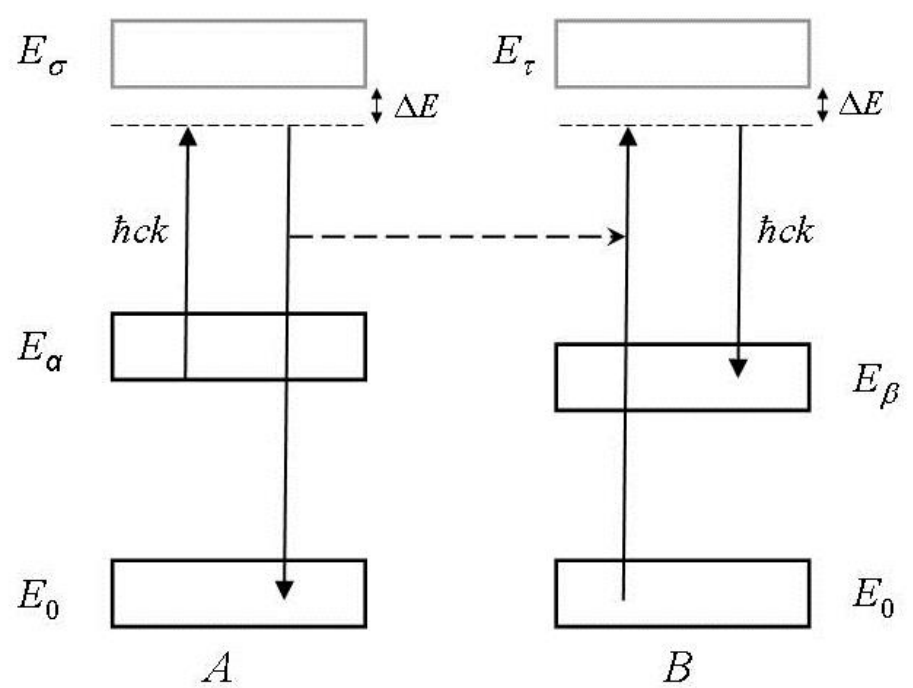

Figure 2. Energetics scheme for OCRET from $A$ to $B$. Solid-head arrows denote four transitions coupling the donor decay $|\alpha\rangle \rightarrow|0\rangle$ and acceptor excitation $|0\rangle \rightarrow|\beta\rangle$, laser interactions denoted by the photon energies. Dotted lines denote virtual states, the closest real states $\mathrm{E}_{\sigma}$ and $\mathrm{E}_{\tau}$ are offset in energy by $\Delta E$. The dashed arrow signifies energy transfer.

$$
M^{(4)}=\left(\frac{m \hbar c k|\mu|^{4}}{4 \pi \varepsilon_{0}^{2} V r^{3}(\Delta E)^{2}}\right) \sin ^{2} \phi \cos \theta \sin \theta,
$$

Here, $\Delta E=E_{\sigma \alpha}-\hbar \omega^{\prime}=E_{\tau \beta}-\hbar \omega^{\prime}$ is a non-zero energy with a significantly lower value than a transition energy, $r$ is the spatial displacement of $B$ from $A$, and angles $\phi$ and $\theta$ denote the orientations of $\mathbf{e}$ with respect to $\hat{\mathbf{k}}$ and $\hat{\mathbf{i}}$, respectively. It is noteworthy that with $\phi=0^{\circ}, \theta=0^{\circ}$ or $\theta=90^{\circ}$, no energy transfer to the acceptor occurs or, alternatively, with 
$\phi=90^{\circ}$ and $\theta=45^{\circ}$ then the results of equation (2) are maximized. The time-dependent probability, $P(t)$, that the energy transfer process can proceed is secured from the Golden Rule, ${ }^{22}$ here expressible in the general form;

$$
P(t)=\frac{2 \pi \rho}{\hbar} \int_{0}^{t}\left|M^{(2)}+M^{(4)}+\ldots\right|^{2} \mathrm{~d} t,
$$

with higher-order quantum amplitudes rapidly diminishing in magnitude. The second-order contribution $M^{(2)}$ corresponds to spontaneous RET - a null quantity in this case - and $\rho$ is the density of states. Inserting (2) into (3) gives the expression;

$$
P(t)=\frac{4 K C^{2} J^{\prime}}{r^{6}} \sin ^{4} \phi \cos ^{2} \theta \sin ^{2} \theta
$$

where the variables are defined as $K=|\boldsymbol{\mu}|^{4} \rho / 8 \pi \varepsilon_{0}^{2} \hbar, \quad C=|\boldsymbol{\mu}|^{2} / 2 c \varepsilon_{0}(\Delta E)^{2} \quad$ and $\quad J^{\prime}=\int_{0}^{t} I^{2}(t) \mathrm{d} t$, in which $I(t) \equiv n \hbar c^{2} k / V$ is the laser irradiance. This concludes the development of fundamental electrodynamic theory for the OCRET mechanism in terms of a donor-acceptor pair. With the results of this analysis, a study into a realistic all-optical switching system can now proceed.

\section{SQUARE-PACKED CONFIGURATION}

One proposed structure for an all-optical system, exploiting the OCRET mechanism, is based on the deposition of thin films on the juxtaposed surfaces of a pair of parallel substrates, where each film contains an ordered set of nanoparticles (henceforth termed pixels); one film comprises donor pixels, and the other, acceptors. First, we investigate a system where the pixels within the adjacent thin-films have a square-packed configuration with lattice constant $l$; within each film the pixels are equally spaced and identical (Fig. 3). It is assumed that each array lies on an ( $x, y)$ plane. The configuration is constructed such that each constituent pixel within the donor film directly corresponds to a counterpart within the acceptor film; these pairs are coded by the coordinates $u$ and $v$ (integer values) and are displaced, one from each other, by $r \hat{\mathbf{k}}$. Furthermore, all nanoparticles in the donor and acceptor films are orientated in the $\hat{\mathbf{i}}$ and $\hat{\mathbf{j}}$ directions, respectively, so that each facing pair satisfies the mutually orthogonal triad condition introduced in the previous Section.

It is expedient to focus on processes by means of which energy can transfer from any one specific excited donor (for convenience located at $u=0, v=0$ ) to any other pixel. First, consider energy relocation to an arbitrary pixel within the acceptor film. Förster transfer is now no longer completely excluded (for acceptor coordinates $u \neq 0, v \neq 0$ ) and a corresponding expression must be included in any $P(t)$ equation for the net transfer probability presented by a square-packed configuration. The appropriate Förster-based expression is derived with use of previous work, ${ }^{23}$ and is given by;

$$
M^{(2)}=\left(\frac{|\mu|^{2}}{4 \pi \varepsilon_{0} l^{3}}\right)\left(\frac{3 u v}{\left(u^{2}+v^{2}+r^{\prime 2}\right)^{\frac{5}{2}}}\right)
$$

Here, $r^{\prime}=r / l$ is the aspect ratio. The transfer contribution due to OCRET is similar to equation (2), and is expressible as; 


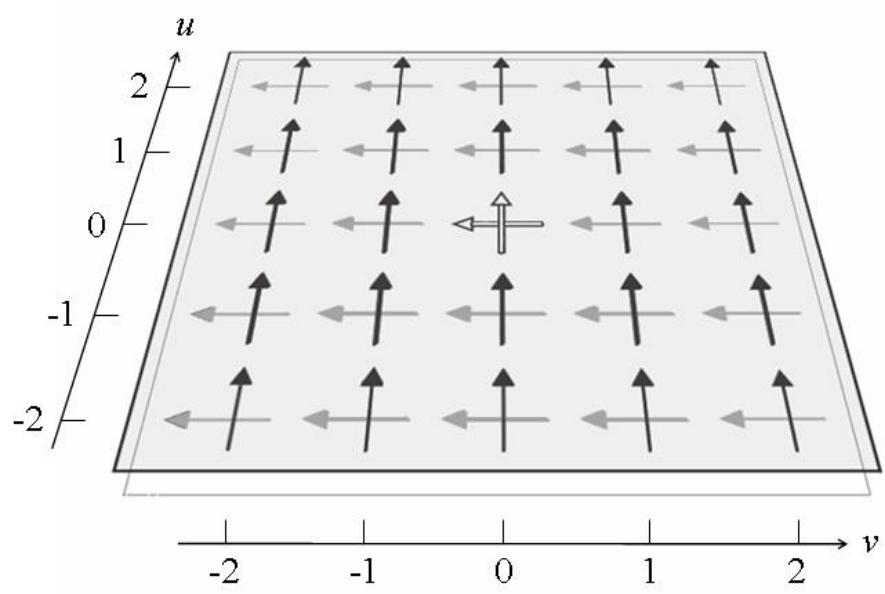

Figure 3. Structure of the square-packed configuration (viewed from above). Both substrates lie in the $(x, y)$-plane, with all donor transition moments (black) in the upper film parallel to the $x$-axis, and all acceptor transition moments (gray) in the lower film parallel to the $y$-axis. The open arrows represent one excited donor and its counterpart acceptor.

$$
M^{(4)}=\left(\frac{-m \hbar c k|\boldsymbol{\mu}|^{4}}{8 \pi \varepsilon_{0}^{2} l^{3} V(\Delta E)^{2}}\right)\left(\frac{u^{2}+v^{2}-2 r^{\prime 2}}{\left(u^{2}+v^{2}+r^{\prime 2}\right)^{\frac{5}{2}}}\right) \sin ^{2} \phi \cos \theta \sin \theta,
$$

Employing equations (5) and (6) with (3), the following is determined;

$$
\begin{aligned}
P(t) & =\frac{K}{l^{6}\left(u^{2}+v^{2}+r^{\prime 2}\right)^{5}}\left[9 u^{2} v^{2} t+6 C J u v\left(u^{2}+v^{2}-2 r^{\prime 2}\right) \sin ^{2} \phi \cos \theta \sin \theta\right. \\
& \left.+C^{2} J^{\prime}\left(u^{2}+v^{2}-2 r^{\prime 2}\right)^{2} \sin ^{4} \phi \cos ^{2} \theta \sin ^{2} \theta\right] .
\end{aligned}
$$

where $J^{\prime}=\int_{0}^{t} I^{2}(t) \mathrm{d} t, I$ being the irradiance of the input laser beam.

Having secured this result, it is also necessary to consider cases of energy transfer from one initially excited donor pixel to another within the same (donor) film. The resulting expression is analogous to equation (7), but with $r^{\prime}$ taken as zero. In the following, we also take the same value of $K$ for such intra-film transfer, recognizing it as marking an upper limit on the extent to which in-film transfer might compromise the sought transfer to the acceptor film. In practice the value will likely be significantly lower. The reason is that the initial excitation of any single donor will usually be followed by rapid intramolecular decay to the energetically lowest part of the excited state continuum, before energy transfer ensues. Consequently the density of states for any donor film pixel acting as acceptor of the energy exchange will be small. By contrast, the usual spectroscopic gradient ${ }^{25}$ that operates between the (necessarily chemically different) donor and acceptor particles will generally ensure energy delivery into a high-density part of the excited state continuum.

To quantify the cross-film transfer we now compare the efficiency of direct energy relocation, from an excited donor to its designated partner in the acceptor film (sought destination, $S$ ), to the summed efficiencies for transfer to any other pixels within either array (cross-talk destinations, $C$ ). The dependence of the result on the aspect ratio is determined and graphically depicted as plots of $P(t)$ for different transfer destinations, as shown in Figs 4 and 5 . Performing the calculations for these graphs, typical parameter values are employed, namely: $\Delta E=3 \times 10^{-20} \mathrm{~J},|\mu|=1$ $\times 10^{-29} \mathrm{C} \mathrm{m}, \phi=90^{\circ}$ and $\theta=45^{\circ}$ (the optimum angles). It transpires that transfer above $|u|,|v|=10$ proves negligible. 
It is notable that the values ascribed to the variables affect the absolute transfer efficiency, but not the destination of the excitation. The fidelity, $F=S /(S+C)$, denotes the fraction of the transfer that delivers energy to the counterpart acceptor; this is plotted on a logarithmic scale and shown in Figs 4 and 5 (insets). The laser input, assumed to be time-independent, has values of $1 \times 10^{12} \mathrm{~W} \mathrm{~cm}^{-2}$ and $1 \times 10^{10} \mathrm{~W} \mathrm{~cm}^{-2}$ for Figs 4 and 5 , respectively. It is clear that the intensity is a major factor in determining the destination of the donor excitation. To achieve transfer losses no greater than $0.2 \mathrm{~dB}$ the aspect ratio, $r^{\prime}$, can be little over 0.3 for $10^{12} \mathrm{~W} \mathrm{~cm}^{-2}$, or 0.06 for $10^{10} \mathrm{~W} \mathrm{~cm}^{-2}$ input - both $r^{\prime}$ values are practicable. The next Section will determine whether improvements are possible with a hexagonal-packed configuration.

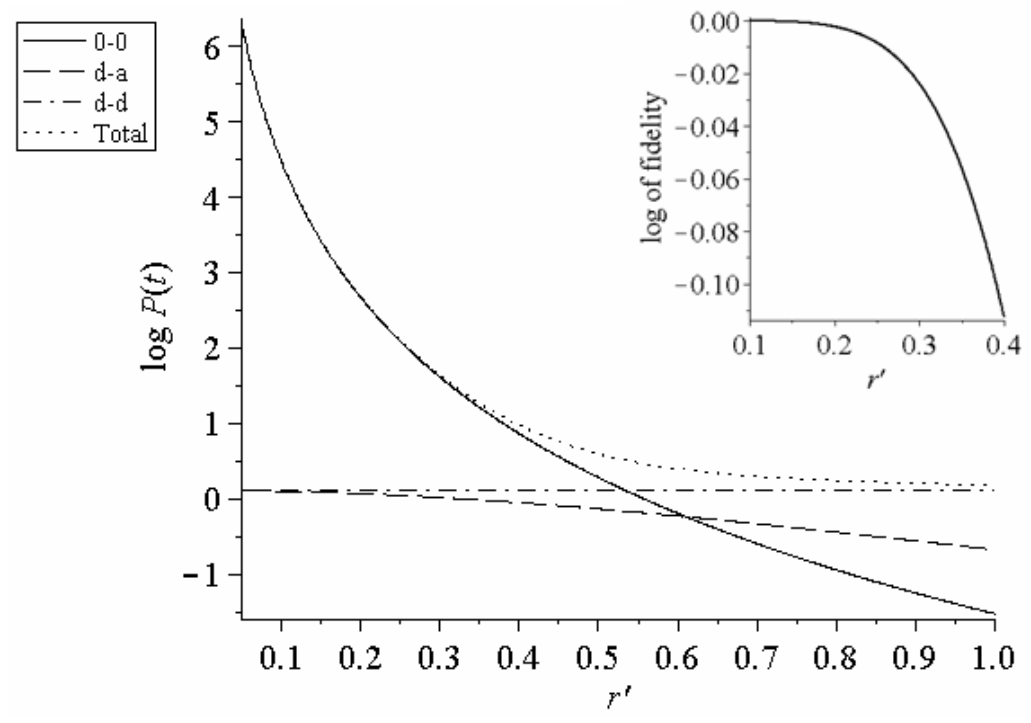

Figure 4. Plot of $\log P(t)$, where $P(t)$ is the time-dependent probability, against the aspect ratio, $r^{\prime}$, for optical transfer from an excited particle in the donor square-packed film to the required destination in the acceptor square film $(0,0)\left(I=10^{12} \mathrm{~W} \mathrm{~cm}^{-2}\right)$; also depicted are the 'cross-talk' probabilities for transfer to another particle in either the acceptor (d-a) or the donor (d-d) film, and the sum of all three transfer possibilities (total). Inset: difference between logarithms of the 0-0 and the sum probabilities for various $r^{\prime}$, signifying on a logarithmic scale the transfer fidelity; on the ordinate axis, each -0.01 increment corresponds to $0.1 \mathrm{~dB}$ loss.

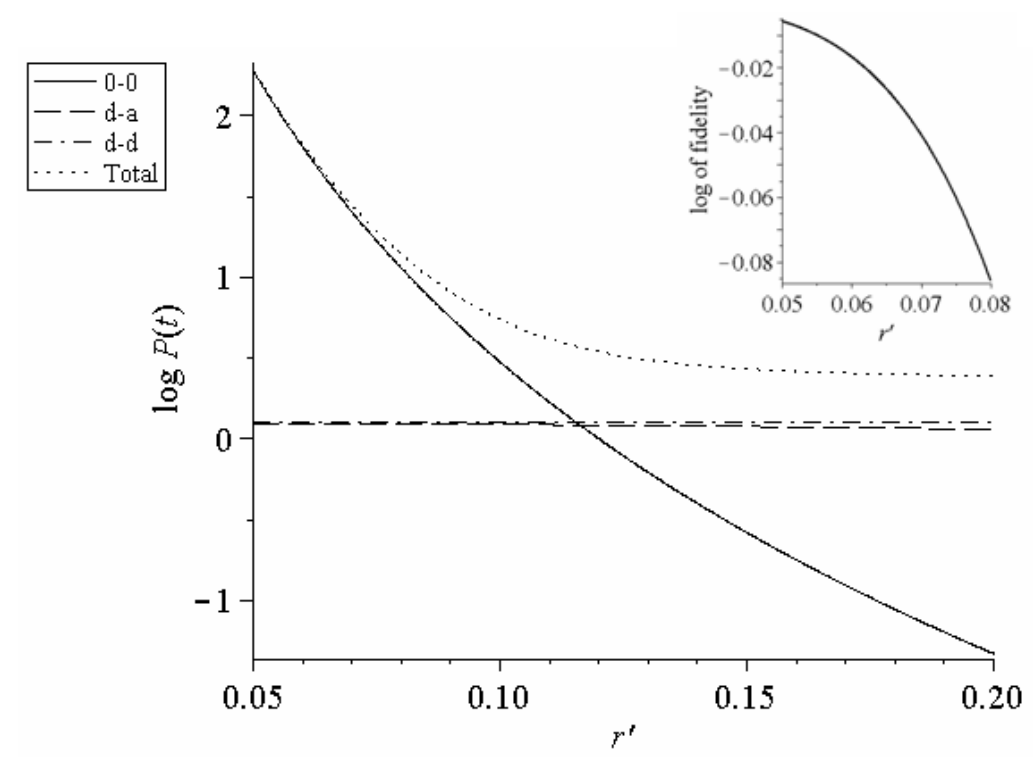

Figure 5. Graph as Fig. 4, but for $I=10^{10} \mathrm{~W} \mathrm{~cm}^{-2}$. 


\section{HEXAGONAL-PACKED CONFIGURATION}

Since a hexagonal-packed configuration is now considered for the thin-film system, it is expedient to choose the new coordinate system illustrated by Fig. 6. For calculations on this system, the donor-acceptor displacement vector $\mathbf{R}=u l \hat{\mathbf{a}}_{1}+v l \hat{\mathbf{a}}_{2}+r \hat{\mathbf{k}}$, cast in terms of the non-orthogonal lattice unit vectors, is converted into Cartesian form so that;

$$
\mathbf{R}=\frac{\sqrt{3} l}{2}(u-v) \hat{\mathbf{i}}+\frac{l}{2}(u+v) \hat{\mathbf{j}}+r \hat{\mathbf{k}}
$$

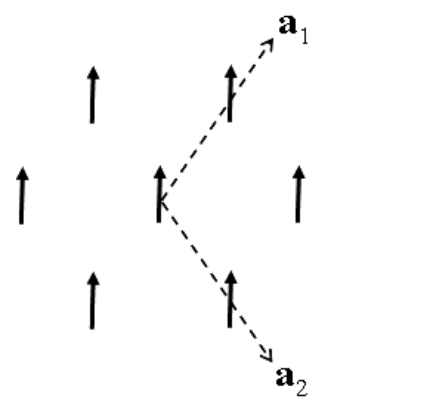

Figure 6. In-plane coordinate system for the hexagonal-packed configuration.

In this system, spontaneous RET is possible from the donor to all other pixels (except where $u=v$ or $u=-v$ ) as is determined from the following expression;

$$
M^{(2)}=\left(\frac{\sqrt{108}|\mu|^{2}}{\pi \varepsilon_{0} l^{3}}\right)\left(\frac{(u-v)(u+v)}{\left(3(u-v)^{2}+(u+v)^{2}+4 r^{\prime 2}\right)^{5 / 2}}\right) .
$$

The transfer contribution due to OCRET is resolved by replicating the previously established procedure, and hence;

$$
M^{(4)}=\left(\frac{-m \hbar c k|\boldsymbol{\mu}|^{4}}{\pi \varepsilon_{0}^{2} l^{3} V \Delta E_{A} \Delta E_{B}}\right)\left(\frac{3(u-v)^{2}+(u+v)^{2}+r^{\prime 2}}{\left(3(u-v)^{2}+(u+v)^{2}+4 r^{\prime 2}\right)^{\frac{5}{2}}}\right) \sin ^{2} \phi \cos \theta \sin \theta
$$

Through use of (9) and (10), with (3), the following result is found:

$$
\begin{aligned}
P(t)= & \frac{K}{l^{6}}\left[1728(u-v)^{2}(u+v)^{2} t+384 \sqrt{3} C J(u-v)(u+v)\left(3(u-v)^{2}+(u+v)^{2}+r^{\prime 2}\right) \sin ^{2} \phi \cos \theta \sin \theta\right. \\
& \left.+64 C^{2} J^{\prime}\left(3(u-v)^{2}+(u+v)^{2}+r^{\prime 2}\right)^{2} \sin ^{4} \phi \cos ^{2} \theta \sin ^{2} \theta\right]\left(3(u-v)^{2}+(u+v)^{2}+4 r^{\prime 2}\right)^{-5} .
\end{aligned}
$$

In the same manner as previously, various plots of $P(t)$ are constructed (Figs 7 and 8). On comparing these graphs it is again evident that the laser intensity plays a major role, as for the case of the square-packed configuration. To achieve transfer losses no more than $0.2 \mathrm{~dB}$ in the present case, $r^{\prime}$ cannot be much greater than 0.11 for an intensity $1 \times 10^{12} \mathrm{~W} \mathrm{~cm}^{-2}$, or 0.025 for $1 \times 10^{10} \mathrm{~W} \mathrm{~cm}^{-2}$ - values that are not particularly favorable in comparison to the previous square-packed system. This is explained by the fact that, in the case of the hexagonal packing, the critical region close to the initially excited donor pixel has a greater number of potential acceptors permitting direct Förster transfer. 


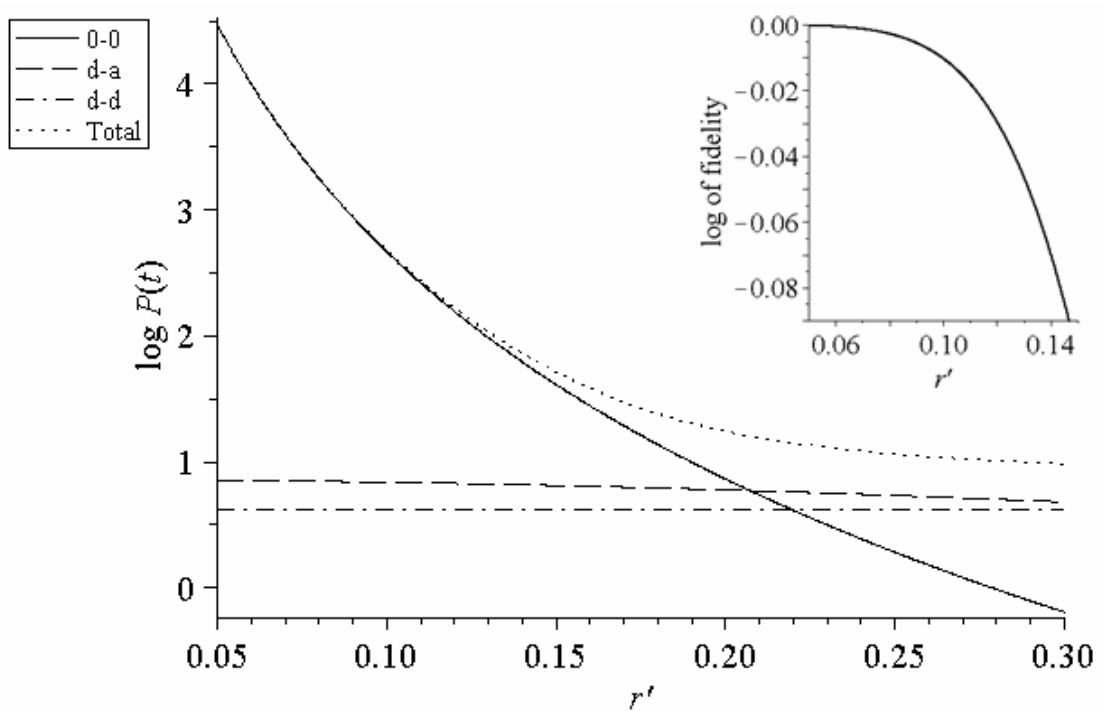

Figure 7. Graph illustrating $\log P(t)$ against $r^{\prime}$ for a pair of hexagonal-packed films. $\left(I=10^{12} \mathrm{~W} \mathrm{~cm}{ }^{-2}\right)$

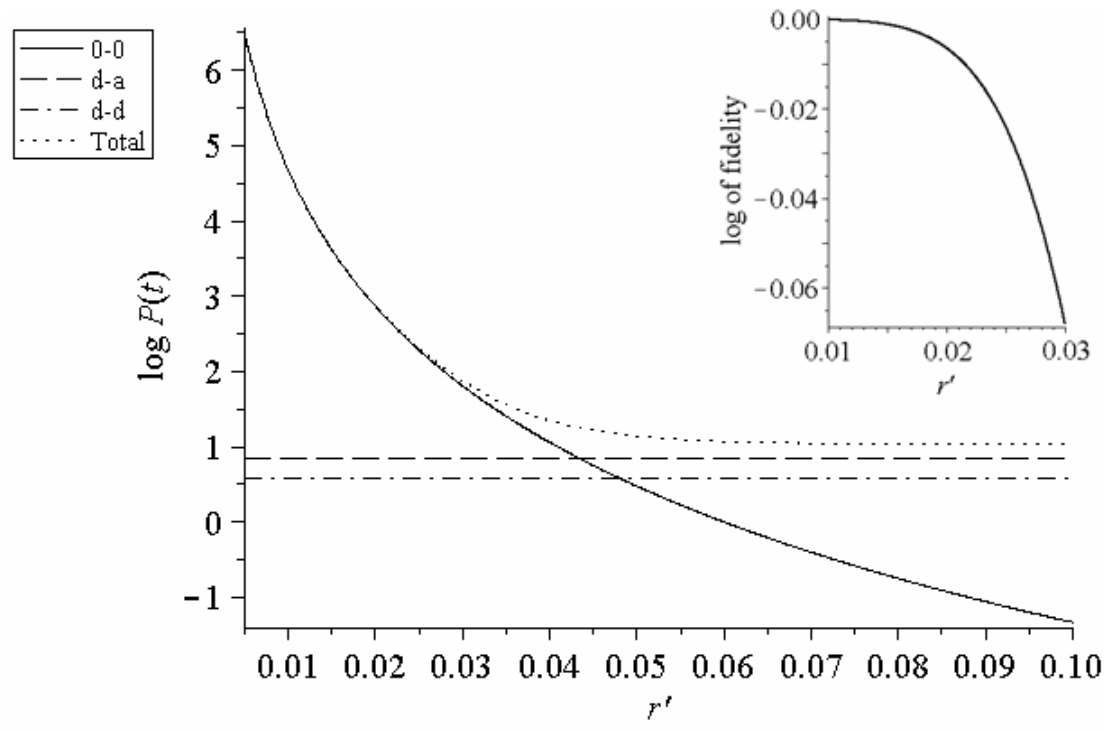

Figure 8. Graph as Fig. 7, but for $I=1 \times 10^{10} \mathrm{~W} \mathrm{~cm}^{-2}$.

\section{DISCUSSION}

The foregoing analysis has addressed the necessary criteria and effective constraints surrounding the possible deployment of OCRET as a means of achieving all-optical switching. The proposed device architecture for such a switch involves thin-film deposits on parallel substrates - an ordered set of nanoparticles being contained within these films. The concept of transfer fidelity, signifying the accuracy of mapping input to designated output, has been introduced and its key determinants identified from the form of the calculated results. Two packing configurations for the nanoparticles have been considered; it is found that a pair of opposing square-packed configurations appears to offer the best prospects for implementation. The detailed analysis has shown that it is possible, by judicious choice of the relative values of the array spacing and lattice constant, together with the levels of laser intensity, to achieve arbitrarily low amounts of cross-talk. The laser pulse length will, in general, significantly exceed the timescale for the OCRET process and, therefore, it is reasonable to assume that the irradiance is effectively constant over time. 
Generally, it may prove expedient to incorporate a suitably transparent ultra-thin spacer material as a separator between the donor and acceptor film layers. The results will be identical to those presented here, except all quantum amplitudes will be multiplied by the appropriate Lorenz factor $\left(n^{2}+2\right) / 3$ - the symbol $n$ denoting the refractive index of the spacer material. Therefore, the absolute transfer efficiency will be enhanced by the inclusion of an ultra-thin spacer with suitable dielectric properties, although the directedness of the excitation delivery is unaltered by such a set-up. The deposition and tailoring of the nanoparticle components, in each active layer, is expected to be achieved by photolithography. ${ }^{26,27}$ Through a novel form of this technique, the nanofabrication of structures similar to those envisaged have already been constructed. ${ }^{28}$ Therefore, there is much promise that a workable device could be manufactured. In terms of applications, the achievement of optical switching in an extensive parallel-processing unit introduces a number of potential applications, beyond simple switching. Logic gate construction - an example of which is given by ref. 29 - is an obvious possibility; the responsiveness to input modulation also suggests other forms of action, possibly leading to optical transistor configurations. Our results furthermore signify that, for example, pixel-based images, written by donor excitation, could be transferred with high fidelity to an acceptor film. In the realm of optical communications, possibilities might be built on the obvious capacity of such a system to act as an ultrafast information buffer; the high level of interest in such devices has already prompted others to explore 'slow-light' methods, where a host of more problematic limitations apply. ${ }^{30,31}$ The systems we have presented offer numerous advantages: viable operation at short uv/visible wavelengths; the obviation of non-standard, expensive optical elements; the lack of any susceptibility to saturation problems; wide applicability to a host of molecular systems; a high information density optimized by using a single donor-acceptor pair for each bit of information; ultrafast response with high repetition rate, high efficiency, and nanoscale miniaturization.

\section{ACKNOWLEDGEMENTS}

We wish to thank the Leverhulme Trust for providing the financial support for this research.

\section{REFERENCES}

1. R. Akimoto, B. S. Li, K. Akita, and T. Hasama, "Ultrafast intersubband optical switching in II-VI-based quantum well for optical fiber communications", Phys. Stat. Sol. (b) 243, pp. 805-812, 2006.

2. Y. Li, A. Bhattacharyya, C. Thomidis, T. D. Moustakas, and R. Paiella, "Nonlinear optical waveguides based on nearinfrared intersubband transitions in GaN/AIN quantum wells", Opt. Express 15, pp. 5860-5865, 2007.

3. G. W. Cong, R. Akimoto, K. Akita, T. Hasama, and H. Ishikawa, "Low-saturation-energy-driven ultrafast all-optical switching operation in (CdS/ZnSe)/BeTe intersubband transition”, Opt. Express 15, pp. 12123-12130, 2007.

4. Y. Fedoryshyn, P. Strasser, P. Ma, F. Robin, and H. Jäckel, "Optical waveguide structure for an all-optical switch based on intersubband transitions in InGaAs/AlAsSb quantum wells”, Opt. Lett. 32, pp. 2680-2682, 2007.

5. P. Bermel, A. Rodriguez, S. G. Johnson, J. D. Joannopoulos, and M. Soljacic, "Single-photon all-optical switching using waveguide-cavity quantum electrodynamics", Phys. Rev. A 74, 043818, 2006.

6. C. Y. Wang, Y. F. Chen, S. C. Lin, W. H. Lin, P. C. Kuan, and I. A. Yu, "Low-light-level all-optical switching", Opt. Lett. 31, pp. 2350-2352, 2006.

7. J. P. Zhang, G. Hernandez, and Y. F. Zhu, "All-optical switching at ultralow light levels", Opt. Lett. 32, pp. 13171319, 2007.

8. M. F. Yanik, S. H. Fan, M. Soljacic, and J. D. Joannopoulos, "All-optical transistor action with bistable switching in a photonic crystal cross-waveguide geometry”, Opt. Lett. 28, pp. 2506-2508, 2003.

9. N. Moll, R. Harbers, R. F. Mahrt, and G. L. Bona, "Integrated all-optical switch in a cross-waveguide geometry", Appl. Phys. Lett. 88, 171104, 2006.

10. M. R. Singh and R. H. Lipson, "Optical switching in nonlinear photonic crystals lightly doped with nanostructures", J. Phys. B 41, 015401, 2008.

11. C. Janke, J. G. Rivas, P. H. Bolivar, and H. Kurz, "All-optical switching of the transmission of electromagnetic radiation through subwavelength apertures”, Opt. Lett. 30, pp. 2357-2359, 2005.

12. G. A. Wurtz, R. Pollard, and A. V. Zayats, "Optical bistability in nonlinear surface-plasmon polaritonic crystals", Phys. Rev. Lett. 97, 057402, 2006. 
13. D. E. Chang, A. S. Sorensen, E. A. Demler, and M. D. Lukin, "A single-photon transistor using nanoscale surface plasmons", Nature Physics 3, pp. 807-812, 2007.

14. Y. H. Huang, S. T. Wu, and Y. Y. Zhao, "All-optical switching characteristics in bacteriorhodopsin and its applications in integrated optics", Opt. Express 12, pp. 895-906, 2004.

15. S. Roy, P. Sharma, A. K. Dharmadhikari, and D. Mathur, "All-optical switching with bacteriorhodopsin”, Opt. Comm. 237, pp. 251-256, 2004.

16. J. Topolancik and F. Vollmer, "All-optical switching in the near infrared with bacteriorhodopsin-coated microcavities”, Appl. Phys. Lett. 89, 184103, 2006.

17. D. L. Andrews and A. A. Demidov, eds: Resonance Energy Transfer (Wiley, Chichester, 1999).

18. P. Allcock, R. D. Jenkins and D. L. Andrews, "Laser assisted resonance energy transfer", Chem. Phys. Lett. 301, pp. 228-234, 1999.

19. P. Allcock, R. D. Jenkins and D. L. Andrews, "Laser-assisted resonance-energy transfer”, Phys. Rev. A 61, 023812, 2000.

20. D. L. Andrews and R. G. Crisp, “Optically activated energy transfer: array implementation”, J. Opt. A 8, pp. S106S112, 2006.

21. D. S. Bradshaw and D. L. Andrews, "Optically controlled resonance energy transfer: Mechanism and configuration for all-optical switching”, J. Chem. Phys. 128, 144506 (2008).

22. E. Fermi, Nuclear Physics (Chicago, University of Chicago Press, 1950).

23. G. J. Daniels, R. D. Jenkins, D. S. Bradshaw and D. L. Andrews, "Resonance energy transfer: The unified theory revisited", J. Chem. Phys. 119, pp. 2264-2274, 2003.

24. D. S. Bradshaw and D. L. Andrews, "The optical control of electronic energy transfer through single and dual auxiliary beams", Proc. SPIE 6988, 698800, 2008

25. D.L. Andrews and J. Rodríguez, "Resonance energy transfer: Spectral overlap, efficiency, and direction", J. Chem. Phys. 127, 084509, 2007.

26. B. D. Gates, Q. Xu, M. Stewart, D. Ryan, C. G. Willson and G. M. Whitesides, "New approaches to nanofabrication: Molding, printing, and other techniques", Chem. Rev. 105, pp. 1171-1196, 2005.

27. R. Garcia, R. V. Martinez and J. Martinez, "Nano-chemistry and scanning probe nanolithographies", Chem. Soc. Rev. 35, pp. 29-38, 2006.

28. W. Wu, D. Dey, O. G. Memis, A. Katsnelson, H. Mohseni "A novel self-aligned and maskless process for formation of highly uniform arrays of nanoholes and nanopillars", Nanoscale Res. Lett. 3, pp. 123-127, 2008.

29. E. K. L. Yeow and R. P. Steer, "Energy transfer involving higher electronic states: a new direction for molecular logic gates", Chem. Phys. Lett. 377, pp. 391-398, 2003.

30. R. W. Boyd and P. Narum, "Slow- and fast-light: fundamental limitations", J. Mod. Opt. 54, pp. 2403-2411, 2007.

31. K. L. Tsakmakidis, A. D. Boardman, and O. Hess, “'Trapped rainbow' storage of light in metamaterials", Nature 450, pp. 397-401, 2007. 